\title{
Is it safe to use propofol in the emergency department? A randomized controlled trial to compare propofol and midazolam
}

\author{
Nik Hisamuddin Nik Ab Rahman • Ahmad Hashim
}

Received: 23 September 2009/Accepted: 14 January 2010/Published online: 25 March 2010

(C) Springer-Verlag London Ltd 2010

\begin{abstract}
Background This study examined the safety and effectiveness of the procedural sedation analgesia (PSA) technique carried out in the emergency department (ED) of a university hospital over a period of 1 year. The research was done to compare the effectiveness and efficacy of moderate sedation of fentanyl combined with either midazolam or propofol for any brief, intense procedure in the ED setting.

Aims The objectives were to observe the occurrence of adverse events in subjects undergoing PSA for intense and painful procedures in the emergency department and to implement the use of capnography as a method of monitoring the patients when they were under PSA.

Methods Forty patients were selected for this study. They were randomly divided into two equal groups using the computer-generated random permuted blocks of four patients. Twenty patients were grouped together as group A and the remaining 20 patients as group B. Drugs used were single blinded to prevent any bias. Drug A was propofol and fentanyl, while drug B was midazolam and
\end{abstract}

The views expressed in this paper are those of the author(s) and not those of the editors, editorial board or publisher.

N. H. N. A. Rahman

Consultant and Head of Emergency Medicine, PPSP, USM,

Kubang Kerian, Malaysia

A. Hashim

Department of Emergency and Trauma, Queen Elizabeth Hospital,

Kota Kinabalu, Sabah, Malaysia

N. H. N. A. Rahman $(\square)$

Department of Emergency Medicine, School of Medical Sciences,

USM,

Kubang Kerian 16150, Malaysia

e-mail: nhliza@hotmail.com fentanyl. The procedures involved included orthopedic manipulation such as reduction of fractures, reduction of dislocated joints, abscess drainage, wound debridement, laceration wound repair and cardioversion. All of the subjects were monitored for their vital signs and end tidal carbon dioxide level every $10 \mathrm{~min}$ till the PSA was completed. The duration of stay in the ED was documented when the subjects had completed the procedure and were released from the department.

Result Of the study population, $75.6 \%$ were males. The mean age was 37.8 years $(95 \%$ CI $33.2,39.8)$. None of the patients developed any major complications while under PSA. The vital signs pre-, intra- and post-procedure were not significantly different in either the propofol or mizadolam groups ( $p$ value $>0.05$ ).

Conclusion This study had proven that there was no difference in adverse event occurrence between the studied drugs during PSA. Propofol can be recommended for use in PSA if the operator is well trained and familiar with the drug.

Keywords Procedural sedation analgesia $\cdot$ Midazolam

Propofol · Emergency department

\section{Introduction}

Patients who attend the emergency department (ED) for any form of trauma and critically ill conditions frequently present with physical or mental pain and agitation. These stresses may be associated with tremendous neuro-humoral elevation of plasma catecholamine, cortisol, glucose, antidiuretic hormone and acute phase protein levels $[1,2]$. This elevation can cause significant tachycardia, hypertension, vasoconstriction, increase oxygen consumption, blunting of 
immune response, and salt and water retention. In addition, these patients are extremely anxious. During these critical situations, procedures may be indicated that require patients to be subjected to some form of chemical induction to facilitate the procedure planned either to save their lives or salvage the remaining functioning organs or limbs. A collective decision needs to be made to choose the most appropriate form of chemical induction for the purpose of analgesia or sedation; usually patients receive the latter [3, 4]. The superiority of one of these drugs and the lack of potentially dangerous adverse reactions would determine the appropriate choice in the ED setting. The main goal of procedural sedation and analgesia (PSA) is to give patients some relief from both pain and anxiety with minimal adverse events. This technique has to be effective in reducing the stress response and improving patient compliance with a procedure [5-7].

Commonly benzodiazepine, such as diazepam or midazolam, is used as an agent for the PSA. Pharmacologically, it takes up to $45 \mathrm{~min}$ for the patients to recover fully and be discharged, and adverse events such as respiratory depression are a known occurrence $[8,9]$. This study examined an alternative agent to PSA, namely propofol. We already know that propofol has a similar mode of action to benzodiazepine, but with a much shorter duration of action [10]. The use of an effective short-acting drug in PSA relieves patients from numerous unpleasant side effects that are commonly seen with the use of conventional long-acting diazepam. We conducted a randomized controlled trial to compare the adverse events between propofol and midazolam during PSA in adult patients who attended the emergency department. The study was approved by the University Hospital Ethics and Research Committee.

\section{Objectives}

The purpose of the study was to compare the outcomes between a combination of fentanyl and propofol with fentanyl and midazolam on subjects during and after PSA.

The outcome measurements were:

1. Vital signs:

a. Systolic and diastolic blood pressure

b. Respiratory rate

c. Heart rate

d. Mean arterial pressure (MAP)

e. Oxygen saturation $\left(\mathrm{SPO}_{2}\right)$

2. End tidal carbon dioxide $\left(\mathrm{EtCO}_{2}\right)$ : to implement microstream capnography during PSA as a method of continuous cardiorespiratory monitoring for adverse event occurrence.

\section{Research hypothesis}

1. Null hypothesis

The use of combined fentanyl and propofol has no difference in adverse event occurrence over fentanyl and midazolam for PSA.

\section{Population}

Patients who fulfill the criteria for PSA

\section{Intervention}

Administration of either fentanyl with midazolam or fentanyl with propofol

\section{Methodology}

Study design

This was a randomized single-blinded control trial carried out in the ED of a university hospital over a period of 12 months. The hospital is a regional tertiary referral center with an attendance rate to the emergency department exceeding 60,000 per year. It is also a teaching hospital responsible for both undergraduate student and residencybased training in many specialized fields including emergency medicine. The proposal for this study was presented to the Department Review Board and Hospital Ethics Committee, and we received approval to proceed. The reference for the certificate of approval is USMKK/PPSP ${ }^{\circledR} /$ JK P\&E. A short-term grant was also approved for conducting the study.

All 40 patients were selected by convenience sampling, and further randomization into two groups was carried out by using the computer-generated random permuted blocks of four patients. All patients recruited did not know which drug they would receive. Each group would receive either drug A or drug B. The drugs used were single blinded. They were supplied by the pharmacy department, wrapped individually and placed in an envelope. Each envelope was sealed and labeled accordingly as drug A or drug B. The operators, emergency physicians and residents in Emergency Medicine, including the main researcher, were unaware of the exact drug to be given until the envelope was opened. Administration of the medications to the patients required precise calculation of the dosage based on the subject's body weight.

Inclusion criteria

All adult patients who presented to the Emergency Department for either brief or intensely painful procedures, 
having marked anxiety or requiring some degree of immobilization were carefully evaluated for suitability to be recruited in this pilot study. The suitability of the subjects was evaluated based on the following criteria:

1. All trauma (except head injury) and non-trauma adult patients

2. All adult patients who were able to give verbal and written consent to participate in the study.

3. All patients who were indicated to have procedural sedation.

4. All patients with physical status of ASA I and II

Patients were numbered and matched to the drugs under study. Detailed explanations regarding the study and the drugs to be used for PSA were provided to all subjects before getting both verbal and written consent. The explanations given included the effects and the side effects of the drugs under study. Patients also received explanations about the procedure that would take place during the PSA. Both verbal and written consents were obtained as required by the University Research Ethical Committee. Standard monitoring (pre-, intra- and post-procedure) of systolic and diastolic blood pressure, heart rate, respiratory rate, end tidal carbon dioxide and $\mathrm{O}_{2}$ saturation were carried out on each patient selected to be in the study (parameters for adverse events). In this study we used nasal prong microstream capnography for monitoring the cardiorespiratory status of patients [11]. The capnographic tubing was attached to a Datascope ${ }^{\circledR}$ monitor manufactured in the USA. The machine was calibrated daily by the same investigator. The vital signs were charted in the case report form every 5 to $10 \mathrm{~min}$ despite continuous monitoring of these parameters. All of the selected patients were to be given intravenous normal saline before the procedure at a rate of $500 \mathrm{ml} / 2 \mathrm{~h}$ to avoid the risk of significant hypotension, and this was part of the fasting (nothing by mouth) procedure prior to the procedure.

Patients were randomly allocated to one of the two groups:

1. Group A: Subjects received IV fentanyl $1 \mathrm{mcg} / \mathrm{kg}$ as a titration dose and propofol $1 \mathrm{mg} / \mathrm{kg}$ followed by propofol $0.5 \mathrm{mg} / \mathrm{kg}$ if needed $(\mathrm{n}=20)$.

2. Group B: Subjects received IV fentanyl $1 \mathrm{mcg} / \mathrm{kg}$ as a bolus dose and a titration dose of midazolam $0.1 \mathrm{mg} / \mathrm{kg}$ and $0.1 \mathrm{mg} / \mathrm{kg}$ if needed $(\mathrm{n}=20)$.

A modified Ramsay Scoring System was used to monitor the drug titration to achieve either grade 3 or 4 on the scoring system (Table 1). Vital signs were monitored and recorded every 5-10 min until the procedure ended. Any cardiorespiratory adverse reactions during the procedures were closely observed, documented and managed according to departmental guidelines. The specialists,
Table 1 Modified Ramsay Scoring System

\begin{tabular}{ll}
\hline Score & Response \\
\hline 1 & Anxious or restless or both \\
2 & Cooperative, orientated and tranquil \\
3 & Responding to commands \\
4 & Brisk response to stimulus \\
5 & Sluggish response to stimulus \\
6 & No response to stimulus \\
\hline
\end{tabular}

residents, medical officers and paramedics in the department were given adequate briefing about the study. This included the issues related to the subject recruitment criteria, the techniques to be carried out, vital sign monitoring and documentation, and data collection through a series of presentations and group discussions 2 months prior to the commencement of the study.

For statistical analysis, the non-parametric test was used due to the small sample size and the non-Gaussian distribution. The Mann-Whitney $U$ test was used to compare the two groups.

\section{Results}

Comparison of demographic and other initial parameters for both groups is shown in Table 2. The senior resident (final year residents) in the Emergency Medicine Department and the specialist emergency physicians performed $75 \%(n=30)$ and $25 \%(n=10)$ of the procedures, respectively. None of the patients in either group developed any adverse events during and after the procedures. No significant drops in blood pressure, heart rate, respiratory rate, end tidal $\mathrm{CO}_{2}$ and pulse oximetry were observed during and after the procedures. Even though a few parameters, such as MAP, SBP and DBP, dropped intra-procedure, these values normalized post-procedure, and the changes were statistically insignificant within and between the groups (Tables 3 and 4).

The end tidal carbon dioxide in the propofol group did not show much variation between the pre- and intraprocedural readings. The means $(\mathrm{SD})$ of pre-procedural and intra-procedural end tidal carbon dioxide were 36.05 (6.57) and 37.80 (4.96), respectively. The post-procedural mean (SD) on the other hand was 36.75 (5.46) with the intra-procedural $\mathrm{p}$ value being 0.775 . In the midazolam group, the means (SD) of end tidal carbon dioxide both preprocedure and intra-procedure were 34.45 (7.82) and 35.05 (8.97), respectively $(\mathrm{p}=0.775)$. After the procedure, the mean (SD) of end tidal carbon dioxide was 36.70 (7.20). Similarly, no statistical difference in $\mathrm{ETCO}_{2}$ was found between the two study groups. 
Table 2 Demographic data and vital sign parameters for both treatment groups

\begin{tabular}{|c|c|c|c|}
\hline Demography/vital parameters & Group A (propofol and fentanyl) & Group B (midazolam and fentanyl) & p-value \\
\hline Number of patients & 20 & 20 & $>0.05$ \\
\hline Age (years) & $40.6(95 \%$ CI $38.2,43.3)$ & $35.0(95 \%$ CI $33.2,37.6)$ & $\mathrm{p}=0.424$ \\
\hline \multicolumn{4}{|l|}{ Sex: } \\
\hline Male & 15 & 16 & \multirow[t]{2}{*}{$\mathrm{p}=0.705$} \\
\hline Female & 5 & 4 & \\
\hline Systolic BP (mmHg) & $127.50(95 \%$ CI $120.00,143.75)$ & $130.00(95 \%$ CI $120.00,140.00$ & $\mathrm{p}=0.241$ \\
\hline Diastolic BP (mmHg) & $75.00(95 \%$ CI $70.00,80.00)$ & $70.00(95 \%$ CI $70.00,80.00)$ & $\mathrm{p}=0.093$ \\
\hline Mean arterial pressure $(\mathrm{mmHg})$ & 94.50 (95\% CI 87.10, 100.00) & 93.30 (95\% CI 87.10, 99.17) & $\mathrm{p}=0.541$ \\
\hline Oxygen saturation (\%) & 99.9 (95\% CI 99.80, 100.00) & $100.00(95 \%$ CI $100.00,100.00)$ & $\mathrm{p}=0.286$ \\
\hline End tidal CO2 (mmHg) & $38.50(95 \%$ CI $33.25,40.00)$ & $36.00(95 \%$ CI $33.25,39.00)$ & $\mathrm{p}=0.662$ \\
\hline Heart rate (per minute) & $80.00(95 \%$ CI $71.00,100.00)$ & $80.00(95 \%$ CI $78.00,96.50)$ & $\mathrm{p}=0.776$ \\
\hline Respiratory rate (per minute) & $19.50(95 \%$ CI $18.00,23.50)$ & $18.00(95 \%$ CI $14.00,22.00)$ & $\mathrm{p}=0.334$ \\
\hline
\end{tabular}

\section{Discussion}

The main goal of PSA is to give patients some relief from both pain and anxiety. In addition, this technique has been clinically shown to be effective in reducing the stress response and improving patient compliance with undergoing a procedure. In general, procedural sedation should be accompanied by analgesia simply because analgesia is able to decrease the stressful effect. This could result in a low requirement for sedatives [12].

The use of PSA has generated much interest and debate, although the technique has been widely practiced in many settings that previously were regarded as being in the domain of anesthesiology [13, 14]. PSA offers many advantages. Firstly, patients are able to maintain consciousness while undergoing an unpleasant procedure. Their tolerance of such painful procedures makes them able to cooperate with the care providers, thereby increasing the compliance further. In addition, this technique does not greatly disrupt the patient's daily activities. Upon being discharged from the hospital, the patients can resume their jobs and daily activities within a relatively short period of time with minimal discomfort. The settings found to be of benefit to patients undergoing PSA include procedures in dentistry (dental and oral surgery), radiology, medicine (bronchoscopy, endoscopy, cardiac studies, pacemaker placement) and gynecology (in vitro fertilization). This approach is also being utilized in the outpatient setting [1517]. In the ED, procedural sedation and analgesia have been widely indicated in overtly anxious patients undergoing procedures such as repair of complicated lacerations, reduction of fractures, application of plaster casts, incision and drainage of abscesses and wound care, thus making it a technique of choice.

Despite the promising outcomes and benefits, some precautions are still required. Adverse events such as

Table 3 Comparing propofol and midazolam by SBP, DBP and MAP

\begin{tabular}{|c|c|c|c|c|c|}
\hline Variables & $\begin{array}{l}\text { Propofol } \\
\text { Mean (SD) }\end{array}$ & $\begin{array}{l}\text { Propofol } \\
\text { Median (IQR) }\end{array}$ & $\begin{array}{l}\text { Midazolam } \\
\text { Mean (SD) }\end{array}$ & $\begin{array}{l}\text { Midazolam } \\
\text { Median (IQR) }\end{array}$ & p-value \\
\hline \multicolumn{6}{|c|}{ Systolic blood pressure } \\
\hline Pre-procedure & $134.00(16.27)$ & $127.50(120.00,143.75)$ & $131.50(15.31)$ & $130.00(120.00,140.00)$ & 0.679 \\
\hline Intra-procedure & $115.50(10.50)$ & $120.00(110.00,120.00)$ & $121.00(17.59)$ & $120.00(110.00,133.00)$ & 0.388 \\
\hline Post-procedure & $122.50(13.43)$ & $120.00(111.25,130.00)$ & $120.75(15.25)$ & $120.00(110.00,128.75)$ & 0.608 \\
\hline \multicolumn{6}{|c|}{ Diastolic blood pressure } \\
\hline Pre-procedure & $75.30(9.27)$ & $75.00(70.00,80.00)$ & $75.25(11.53)$ & $70.00(70.00,80.00)$ & 0.731 \\
\hline Intra-procedure & $64.75(8.66)$ & $65.00(60.00,70.00)$ & $65.75(11.62)$ & $67.50(60.00,70.00)$ & 0.868 \\
\hline Post-procedure & $70.25(6.52)$ & $70.00(60.00,78.75)$ & $73.00(15.25)$ & $70.00(61.25,78.75)$ & 0.989 \\
\hline \multicolumn{6}{|l|}{ MAP } \\
\hline Pre-procedure & $94.45(9.88)$ & $94.50(87.10,100.00)$ & $94.66(10.31)$ & $93.30(87.10,99.17)$ & 0.765 \\
\hline Intra-procedure & $82.00(8.70)$ & $81.65(76.70,89.17)$ & $83.84(12.27)$ & $85.00(74.97,89.17)$ & 0.744 \\
\hline Post-procedure & $87.76(9.41)$ & $86.70(80.00,92.90)$ & $87.73(12.44)$ & $86.70(80.82,92.25)$ & 0.733 \\
\hline
\end{tabular}


Table 4 Comparisons of respiratory parameters for both treatment groups

\begin{tabular}{|c|c|c|c|c|c|}
\hline Variables & $\begin{array}{l}\text { Propofol } \\
\text { Mean (SD) }\end{array}$ & $\begin{array}{l}\text { Propofol } \\
\text { Median (IQR) }\end{array}$ & $\begin{array}{l}\text { Midazolam } \\
\text { Mean (SD) }\end{array}$ & $\begin{array}{l}\text { Midazolam } \\
\text { Median (IQR) }\end{array}$ & p-value \\
\hline \multicolumn{6}{|l|}{ Respiratory rate } \\
\hline Pre-procedure & $20.30(4.90)$ & $19.50(18.00,23.50)$ & $18.25(5.00)$ & $18.00(14.00,22.00)$ & 0.574 \\
\hline Intra-procedure & $20.90(5.31)$ & $21.50(16.50,25.00)$ & $18.40(4.60)$ & $18.50(14.50,21.75)$ & 0.082 \\
\hline Post-procedure & $20.10(5.28)$ & $20.00(15.00,24.00)$ & $18.20(3.59)$ & $18.00(16.00,22.00)$ & 0.554 \\
\hline \multicolumn{6}{|l|}{ Oxygen saturation } \\
\hline Pre-procedure & $99.90(0.45)$ & $100.00(100.00,100.00)$ & $99.85(0.49)$ & $100.00(100.00,100.00)$ & 0.226 \\
\hline Intra-procedure & $99.80(0.89)$ & $100.00(100.00,100.00)$ & $99.05(2.01)$ & $100.00(99.25,100.00)$ & 0.106 \\
\hline Post-procedure & $99.90(0.45)$ & $100.00(100.00,100.00)$ & $99.80(0.69)$ & $100.00(100.00,100.00)$ & 0.215 \\
\hline \multicolumn{6}{|l|}{$\mathrm{ETCO}_{2}$} \\
\hline Pre-procedure & $36.05(6.57)$ & $38.50(33.25,40.00)$ & $34.45(7.82)$ & $36.00(33.25,39.00)$ & 0.558 \\
\hline Intra-procedure & $37.80(4.96)$ & $38.00(34.25,42.25)$ & $35.05(8.97)$ & $39.50(25.50,40.75)$ & 0.775 \\
\hline Post-procedure & $36.75(5.46)$ & $38.00(35.00,40.00)$ & $36.70(7.20)$ & $39.00(33.50,42.00)$ & 0.606 \\
\hline
\end{tabular}

cardiorespiratory compromise occurring during and after PSA are commonly reported. Reasons for the complications include inexperienced physicians administering the PSA, improper equipment for monitoring, wrong choice of drugs and presence of comorbidities that lead to the adverse events. Patients undergoing PSA must be closely and continuously monitored to avoid any progression into a deeper state of sedation [18, 19]. Should this occur, the actual purpose of PSA would be nullified. Monitoring can be achieved effectively through visual observation coupled with the use of a pulse oxymeter and a capnograph. The procedure can be carried out safely and non-invasively with the advent of new monitoring strategies. The use of a pulse oxymeter, for example, coupled with non-invasive monitoring of blood pressure optimizes the comfort and care in patients receiving PSA [20].

The pulse oxymeter has been used to monitor the level of oxygenation. Since sedation can result in the emergence of apnea and hypoventilation, failure to detect these conditions may eventually lead to oxygen desaturation. The pulse oxymeter has been clinically shown to be a relevant tool to monitor the existence of oxygen desaturation. The capnograph is another very useful instrument for recognizing any ventilatory and circulatory problems that occur during sedation. A capnograph has the capacity to provide early warnings of apnea and detect the occurrence of respiratory depression, obstruction or laryngopasm through the monitoring of end tidal expiratory carbon dioxide $\left(\mathrm{EtCO}_{2}\right)$, which can be accurately measured. Respiratory depression is said to take place when $\mathrm{O} 2$ saturation is $<90 \mathrm{mmHg}, \mathrm{EtCO}_{2}$ is $>50 \mathrm{mmHg}$ or when there is an absence of EtCO2 waveform [21, 22].

Benzodiazepine such as midazolam has been widely used in many surgical procedures performed under local anesthesia. The use of midazolam has been well docu- mented to enhance patient comfort, improve operating conditions and most importantly, because of its amnesic properties, prevent patients from recalling unpleasant events during the procedure. In addition, midazolam acts indirectly as a gamma amino butyric acid agonist and is relatively cardio-respiratory safe. Once administered, it is rapid in onset and has a short duration of action, which makes midazolam a very popular drug of choice for PSA [23]. However, over the last few years, propofol has emerged quite rapidly as a good agent to be used in PSA by non-anesthesiologists especially in the ED. When given intravenously, the effect is almost immediate. Because of its short half-life, patients on propofol will recover rapidly.

Propofol has a bronchodilating effect, which makes it an appropriate drug of choice for patients with bronchial asthma. Its anti-emetic characteristic gives an added advantage to minimize post-sedation nausea [24]. However, propofol reduces the mean arterial pressure (MAP), which makes it a rather poor choice in patients who develop hypotension, cardiorespiratory compromise or have head injuries. Apnea and painful injection are other disadvantages of this drug. It may also cause deep sedation and analgesia. Deep sedation is said to take place when a purposeful response is triggered with repeated stimuli, while moderate sedation is a purposeful response to light stimuli, e.g., verbal and tactile. Respiratory depression occurs mainly during deep sedation and not moderate sedation. In moderate sedation, the protective airway reflex is intact and thus reduces the risk of aspiration. Respiratory depression occurs in $19 \%$ of patients receiving propofol alone as compared to those who received fentanyl alone (20\%), while a combination of midazolam with fentanyl causes respiratory depression in $23 \%$ of cases. Due to deep or over-sedation produced by propofol within the normal 
range, anesthetists object to its use in the emergency and other departments [25]. However, Zed et al. and Sipe et al. have shown that a bolus dose of $1 \mathrm{mg} / \mathrm{kg}$ followed by $0.5 \mathrm{mg} / \mathrm{kg}$ (when it is necessary) may reduce both the hypoxia and apnea compared to the $1.5 \mathrm{mg} / \mathrm{kg}$ dose $[26,27]$.

This randomized controlled trial study was initiated primarily to determine the safety of PSA delivery in the ED setting. In addition, this study also aimed to evaluate the safety profile and effectiveness of the two sedative hypnotics, namely propofol and midazolam, which have been commonly used in patients undergoing PSA. Nonparametric analysis of data was applied in this study because of the small sample size and non-Gaussian distribution in descriptive analysis. Two tests were applied throughout, namely the Mann-Whitney U test (chi-square) and Kruskal-Wallis (chi-square and $\mathrm{P}$ value). The data were further analyzed as non-stratified to see the effect without the confounder, for example, the sex of subjects in the study and later the stratified analysis where the confounders were controlled. In this study, age could not be stratified because the subjects recruited were adults. This trial, which took 1 year to complete, involved 40 subjects who fully consented to participate. There were no drop-outs. Clear instructions and guidelines were strictly adhered to in order to minimize bias as well as errors in evaluating the safety profile and efficacy between the two drugs used in PSA. A standard set of forms was utilized, and all relevant information required for the study has been clearly documented.

This study did not show remarkable differences between the two drugs used, namely propofol and midazolam in PSA. Both drugs have been found to be safe for use and did not cause any serious adverse side effects, such as hypoventilation, hypotension, apnea, hypoxia or allergic reactions. None of the patients involved in this study developed any complications throughout the process of procedural sedation. These findings were consistent and supported the study done by Hasen et al. on PSA using intravenous propofol for 48 patients undergoing elective orthopedic surgery under regional blockade [28]. They firmly concluded that propofol is a safe and effective drug to be used in PSA with no respiratory or cardiovascular depression, or other undesirable adverse effects. Cheol et al., who conducted a randomized double-blind comparative study using propofol alone and combined propofol and midazolam for colonoscopy, found that both propofol alone and combined propofol and midazolam are safe and effective [29].

However, some results found in this trial remained significant and may illustrate the relevant direction of the future use of these drugs in the emergency department setting.
Changes in blood pressure recorded during procedural sedation

There was a significant and relevant variation in blood pressure before, during and after the procedure. It was found in this study that propofol caused a marked reduction in both the systolic and diastolic blood pressures during the procedure when compared to midazolam. Systolic blood pressure dropped from a mean (SD) of $134.00 \mathrm{mmHg}$ (16.27) to $115.50 \mathrm{mmHg}$ (10.50), while the diastolic blood pressure dropped from a mean (SD) of $75.30 \mathrm{mmHg}(9.27)$ to $65.00 \mathrm{mmHg}(8.66)$ intra-procedure. After the procedure ended, the systolic and diastolic blood pressures normalized to the pre-procedural value (SD) $122.5 \mathrm{mmHg}$ (13.43).

The midazolam group on the other hand showed a marked elevation in both systolic and diastolic blood pressures when the procedure was carried out. Prior to the procedure the mean systolic blood pressure was $121.00 \mathrm{mmHg}$ (17.59) and increased to $131.50 \mathrm{mmHg}$ (15.31) intra-procedure, then normalized with the completion of the procedure. A similar observation was seen in the diastolic blood pressure. The different blood pressure responses can be explained by the different pharmacodynamics of the two drugs. Propofol is known to affect the cardiovascular function of the body system, in particular compromising the blood pressure mainly through the vasodilation effect, whereas benzodiazepine is commonly known to depress more of the respiratory function.

\section{Effects on mean arterial pressure}

In this study both propofol and midazolam did not have a significant effect on the mean arterial pressure when a procedure was carried out to completion. Despite the changes in MAP in both drugs under study, during the procedure the $\mathrm{p}$ value showed non-statistical significance $(p=0.774)$. Both drugs did not result in changes of perfusion to various organs in the body, further confirming the safety of both drugs.

\section{Effects on respiratory rate}

Both propofol and midazolam showed no difference in respiratory rate during and after procedures $(p=0.106)$. This could be due to the appropriate titration dose technique used for the procedural sedation.

\section{Effects on heart rate}

There was a slight reduction of heart rate recorded in this trial during the procedure in the propofol group with a mean value of 76.40 (16.37), while the pre-procedure mean heart rate (SD) was 83.55 (16.3). The heart rate reduced slightly after the completion of the procedure, but did not 
reach the pre-procedural level. In the midazolam group, the heart rate was also found to be reduced during the procedure stage. The mean heart rate during the procedure was 79.65 (16.69) and the mean before the procedure (SD) was 85.45 (13.38). The $\mathrm{p}$ value for both groups was 0.795 .

\section{End tidal carbon dioxide level}

Capnography could provide an accurate assessement of various aspects of respiratory functions, which included real-time ventilatory status, endotracheal tube placement and function, ventilatory circuit disconnection and airway leaks. This study utilized nasal prong microstream devices, which measured respiratory gas concentration remotely by aspirating a small sample of gas from the breathing circuit through tubing to a sensor located inside the monitor. The partial pressure of carbon dioxide was kept constant between the level of $35 \mathrm{mmHg}$ to $45 \mathrm{mmHg}$. Any adverse changes or complications in respiratory functions that arise can be easily detected through capnography. Early detection would ensure an immediate intervention on the patient [30].

In this study, the end tidal carbon dioxide level in the propofol group did not show much variation between the pre- and intra-procedural readings. The means (SD) of preprocedural and intra-procedural end tidal carbon dioxide levels were 36.05 (6.57) and 37.80 (4.96), respectively. The post-procedural mean was 36.75 (5.46) with an intraprocedural $p$ value of 0.775 . In the midazolam group, the mean end tidal carbon dioxide levels both pre-procedure and intra-procedure were 34.45 (7.82) and 35.05 (8.97), respectively $(\mathrm{p}=0.77)$. There were no marked differences in end tidal carbon dioxide readings between these two drugs. This was because the doses of both drugs used were adequately safe, and additional use of capnography monitoring of the end tidal carbon dioxide level could provide early warning should there be evidence of apnea or hypoventilation. Interventions including repositioning of the airway can be carried out if there is evidence of reduction of partial pressure of carbon dioxide. Jennifer et al. pointed out that capnography is capable of providing important data regarding airway permeability, cardiac and circulatory function and ventilator performance, apart from its ability to evaluate alveolar ventilation. The use of capnography could replace the need to analyze ABG in a non-invasive manner [31]. It is important to emphasize that all subjects in both groups A and B were provided with continuous oxygen via nasal prong devices throughout the PSA. In a review done by Vargo, he has stressed the significance of unrecognized respiratory difficulty and hypoxemia as a major factor contributing to morbidity and mortality among patients with PSA in the ambulatory setting. To overcome these potential risks, he strongly recommended the use of capnography, which can monitor and detect any evidence of respiratory compromise early on with the combined use of pulse oxymetry.

In all the domains of recorded vital signs, it was found to be insignificant, as shown by the $p$ value of $>0.005$ on Mann-Whitney $U$ test. For the respiratory rate in male subjects, during the procedure a slight reduction of respiratory rate was observed in the midazolam group from a mean value of 19.37 (4.96) per minute to 19.12 (4.36) $(p=0.148)$. There was no similar reduction among males in the propofol group using stratified Mann-Whitney analysis.

The time interval to discharge for male subjects in the propofol group was observed to be shorter when compared to the midazolam group with a mean (SD) value of 30.33 (12.46) as compared to 59.06 (22.7), respectively, with a $p$ value of 0.001 . In female subjects, the mean (SD) values for the propofol and midazolam were 26.00 (4.18) and 122.50 (28.16), respectively $(\mathrm{p}=0.001)$. There was no significant change found when the data were stratified further to look at the effect of the confounder (sex) on the differences between both propofol and midazolam. Other studies had shown similar patterns of outcome [32, 33].

\section{Limitations of the study}

Several limitations have been identified in this study.

1. Generally the size of the sample recruited was judged to be small. There were reasons for such a small sample gathered. This study was carried out for a period of 1 year as part of the clinical exposure for the completion of the Master program; the sample size was reduced to an acceptable 40 subjects and had received approval from the hospital ethics committee. The subjects were then subdivided equally into two groups comprising 20 subjects each, further reducing the size and thus the power of this study.

2. During the process of administration of the drugs under study, the operators were aware of the drugs that were given to the patients when the envelope was opened. The operator could easily recognize the types of drugs given by the different sizes of ampules, the color of the ampules, the inscription on the ampule and the quantity of the drug in the ampule. This method did not actually protect the anonymity of the drug administered and could possibly have resulted in some level of bias.

3. As a result of the small sample size, the parametric analysis could not be carried out. The data were analyzed using the non-parametric Mann-Whitney U and Kruskal-Wallis analyses.

4. As the main objective of the study was to observe the outcomes of the drugs used-propofol and midazolam 
on vital signs, $\mathrm{EtCO}_{2}$ and duration of regained consciousness - other cofounders like the clinical procedures and number of doses of the drug top-up were not included in the data analysis. for practice for PSA need to be developed to ensure this technique is safe and effective when carried out in the ED.

\section{Recommendations}

This study did not detect any occurrence of hypoxemia, hypoventilation or apnea in any of the participating subjects despite evidence indicating that both propofol and midazolam do cause the above complications. This is because the study used appropriate doses to commence procedural sedation in the emergency department setting. As both of these drugs are safe and effective, the main researcher would like to recommend the following doses to be administered when performing PSA in the ED setting. For propofol, the recommended dose is $1 \mathrm{mg} / \mathrm{kg}$ body weight as a bolus dose followed by $0.5 \mathrm{mg} / \mathrm{kg}$ if required in a titrating dose. For midazolam, the recommended dose is $0.1 \mathrm{mg} / \mathrm{kg}$ body weight as a bolus dose followed by $0.1 \mathrm{mg} / \mathrm{kg}$ in a titrating dose when necessary. Although these drugs are regarded as safe and effective, caution is still needed to ensure avoidance and minimization of any possible complications. Appropriate attention must be given to all patients on PSA by continuous monitoring before, during and after the procedure has been completed. Studies have indicated that the use of supplemental oxygen could significantly reduce the magnitude of oxygen desaturation in any procedure performed when a patient is undergoing procedural sedation. This fact should be considered seriously.

A capnograph is a very useful instrument to monitor the arterial partial pressure of carbon dioxide indirectly during PSA. It is a very sensitive tool for picking up these changes at very early stages of hypoventilation, and its detection accuracy is heavily supported by numerous studies worldwide. It is recommended that capnography should be used when PSA is performed in the ED setting. Early intervention could be instituted once abnormal partial pressure of carbon dioxide is detected.

Finally, all patients planned for PSA in the ED setting must be thoroughly assessed, which involves detailed medical history taking in order to prevent the possibility of developing unexpected complications and delay in recovery after the procedure has been completed.

\section{Conclusions}

Both propofol and midazolam given at the recommended doses are equally safe and effective in PSA performed in the ED setting. However, a protocol and clinical guidelines

\section{References}

1. Finn PJ, Plank LD, Clark MA, Connolly AB, Hill GL (1996) Progressive cellular dehydration and proteolysis in critically ill patients. Lancet 347:654-656

2. Gamrin L, Andersson K, Hultman E, Nilsson E, Essen P, Wernerman J (1997) Longitudinal changes of biochemical parameters in muscle during critical illness. Metabolism 46:756762

3. Bahn EL, Holt KR (2005) Procedural sedation and analgesia: a review and new concepts. Emerg Med Clin North Am 23(2):503-517

4. Meredith JR, O'Keefe KP, Galwankar S (2008) Pediatric procedural sedation and analgesia. J Emerg Trauma Shock 1(2):88-96

5. Bordo D, Chan SB, Shin P (2008) Patient satisfaction and return to daily activities using etomidate procedural sedation for orthopedic injuries. West J Emerg Med 9(2):86-90

6. Symington L, Thakore S (2006) A review of the use of propofol for procedural sedation in the emergency department. Emerg Med J 23(2):89-93, Review

7. Kennedy RM, Luhmann JD, Luhmann SJ (2004) Emergency department management of pain and anxiety related to orthopedic fracture care: a guide to analgesic techniques and procedural sedation in children. Paediatr Drugs 6(1):11-31, Review

8. Di Liddo L, D'Angelo A, Nguyen B, Bailey B, Amre D, Stanciu C (2006) Etomidate versus midazolam for procedural sedation in pediatric outpatients: a randomized controlled trial. Ann Emerg Med 48(4):433-440, 440.e1. Epub 2006 Apr 27

9. Zakko SF, Seifert HA, Gross JB (1999) A comparison of midazolam and diazepam for conscious sedation during colonoscopy in a prospective double-blind study. Gastrointest Endosc 49 (6):684-689

10. Frank LR, Strote J, Hauff SR, Bigelow SK, Fay K (2006) Propofol by infusion protocol for ED procedural sedation. Am J Emerg Med 24(5):599-602

11. Yanagidate F, Dohi S (2006) Modified nasal cannula for simultaneous oxygen delivery and end-tidal $\mathrm{CO} 2$ monitoring during spontaneous breathing. Eur J Anaesthesiol 23(3):257-260

12. Paris $\mathrm{P}$, Yealy $\mathrm{D}$ A procedural sedation and analgesia fasting consensus advisory: one small step for emergency medicine, one giant challenge remaining. Ann Emerg Med 49(4):465-467

13. Godwin SA, Caro DA, Wolf SJ et al (2005) Clinical policy: procedural sedation and analgesia in the emergency department. Ann Emerg Med 45(2):177-196

14. Anesthesiologists Task Force on Sedation and Analgesia by NonAnesthesiologists (2002) Practice guidelines for sedation and analgesia by non-anesthesiologists. Anesthesiology 96(4):10041017

15. Chuang E, Zimmerman A, Neiswender KM, Liacouras CA (2001) Sedation in pediatric endoscopy. Gastrointest Endosc Clin N Am 11(4):569-584

16. Lee H, Kim JH (2009) Superiority of split dose midazolam as conscious sedation for outpatient colonoscopy. World J Gastroenterol 15(30):3783-3787

17. Patatas K, Koukkoulli A (2009) The use of sedation in the radiology department. Clin Radiol 64(7):655-663, Epub 2008 Dec 30. Review 
18. Melendez E, Bachur R (2009) Serious adverse events during procedural sedation with ketamine. Pediatr Emerg Care 25 (5):325-328

19. Nilsson A, Steinvall I, Bak Z, Sjöberg F (2008) Patient controlled sedation using a standard protocol for dressing changes in burns: patients' preference, procedural details and a preliminary safety evaluation. Burns 34(7):929-934, Epub 2008

20. Vargo JJ (2007) Minimizing complications: sedation and monitoring. Gastrointest Endosc Clin N Am 17(1):11-28, v-vi. Review

21. Krauss B, Hess DR (2007) Capnography for procedural sedation and analgesia in the emergency department. Ann Emerg Med 50 (2):172-181, Epub 2007 Jan 12

22. Lightdale JR et al (2006) Microstream capnography improves patient monitoring during moderate sedation: a randomized, controlled trial. Pediatrics 117(6):e1170-e1178, Epub 2006 May 15

23. Demiraran Y (2007) e $t$ al. The comparison of dexmedetomidine and midazolam used for sedation of patients during upper endoscopy: A prospective, randomized study. Can J Gastroenterol 21(1):25-29

24. Green SM, Krauss B. Barriers to propofol use in emergency medicine. Ann Emerg Med. 2008 Oct;52(4):392-8. Epub 2008 Mar 4.

25. Hohl CM, Sadatsafavi M, Nosyk B, Anis AH. Safety and clinical effectiveness of midazolam versus propofol for procedural sedation in the emergency department: a systematic review. Acad Emerg Med. 2008 Jan;15(1):1-8. Review.

26. Sipe BW, Scheidler M, Baluyut A, Wright B (2007) A prospective safety study of a low-dose propofol sedation protocol for colonoscopy. Clin Gastroenterol Hepatol 5(5):563-566

27. Zed PJ, Abu-Laban RB, Chan WW, Harrison DW (2007) Efficacy, safety and patient satisfaction of propofol for procedural sedation and analgesia in the emergency department: a prospective study. CJEM 9(6):421-427

28. Hasen KV, Samartzis D, Casas LA, Mustoe TA (2003) An outcome study comparing intravenous sedation with midazolam/ fentanyl (conscious sedation) versus propofol infusion (deep sedation) for aesthetic surgery. Plast Reconstr Surg 112(6):16831689

29. Cheol et al. (2006) "A comparative study using propofol alone versus propofolcombined midazolam for colonoscopy: randomized double-blind study." Gastroint Endosc 63, No. 5

30. Nagler J, Krauss B (2008) Capnography: a valuable tool for airway management. Emerg Med Clin North Am 26(4):881-897, vii

31. Jenifer Lightdale $H$ et al (2005) "A randomized double-blind controlled trial of microstream capnography to reduce oxygen desaturation during gastrointestinal procedures with conscious sedation." Gastroint Endosc 61 No. 5

32. Bell A, Treston G, Cardwell R, Schabort WJ, Chand D (2007) Optimization of propofol dose shortens procedural sedation time, prevents resedation and removes the requirement for postprocedure physiologic monitoring. Emerg Med Australas 19 (5):411-417

33. Frank LR, Strote J, Hauff SR, Bigelow SK, Fay K (2006) Propofol by infusion protocol for ED procedural sedation. Am J Emerg Med 24(5):599-602

Nik Hisamuddin Nik Ab. Rahman was born in Kota Bharu, Kelantan, Malaysia, on 22 October 1969. He is currently an Associate Professor and Head and Consultant in Emergency Medicine at the School of Medical Sciences, USM, Kota Bharu, Malaysia. He got his Bachelor of Medicine and of Surgery $(\mathrm{MBChB})$ at the University of Glascow, Scotland. He earned his Master of Medicine (MMED) at School of Medical Sciences at Universiti Sains Malaysia, Kubang Kerian, Kelantan, Malaysia. He has received training in Diving and Hyperbaric Medicine at Hyperbaric International, Key Largo, Florida; Nix Medical Center for Hyperbaric \& Wound Care, San Antonio, Texas as a Hyperbaric Medicine \& Diving Medical Officer. Since 2006 he has been a Clinical Fellow in Accident \& Emergency Medicine at the Royal Infirmary of Edinburgh, Scotland, UK. 